\title{
Violence sexuelle en période de guerre: une revue des études empiriques
}

\author{
Louis Paluku Sabuni
}

Ancien Directeur Général à l'Institut Supérieur Panafricain de Santé Communautaire de Bunia (ISPASC-Bunia), Ituri, Province Orientale, et Directeur du Centre de Recherche Interdisciplinaire pour le Développement (CRID) à Beni, Nord-Kivu, R. D. Congo. sbn4you2004@yahoo.co.uk

\section{Bernard Roy}

Centre de Recherche Interdisciplinaire sur la Violence Familiale et la Violence Faite aux Femmes (CRI-VIFF), Faculté des Sciences Infirmières, Université Laval, Québec, Canada.

\section{Jocelyn Lindsay}

Membre du Centre de Recherche Interdisciplinaire sur la Violence Familiale et la Violence Faite aux Femmes (CRI-VIFF) et responsable de l'équipe de recherche Masculinités et Société; Professeur titulaire, École de Service Social, Faculté des Sciences Sociales, Université Laval, Québec, Canada.

\section{Geneviève Lessard}

Codirectrice du Centre de Recherche Interdisciplinaire sur la Violence Familiale et la Violence Faite aux Femmes (CRI-VIFF); Professeur Agrégée, École de Service Social, Faculté des Sciences Sociales, Université Laval, Québec, Canada.

\section{Résumé}

Cet article passe en revue l'état des connaissances sur la violence sexuelle en période de conflit armé, ainsi que les différentes méthodologies utilisées. II examine la littérature théorique et empirique, en utilisant une combinaison de méthodes qualitatives et quantitatives. Les résultats montrent que la violence sexuelle «structurelle » a ses racines dans diverses institutions sociales qui l'exacerbent en la transformant en violence "conjoncturelle » et qui font obstacle à la prévention et la protection des victimes. La violence sexuelle a des conséquences physiques, psychologiques et sociales graves et peut replonger les victimes dans un cycle de violence. Nos travaux suggèrent que la violence sexuelle devra être analysée en période de conflit aussi bien qu'en période de paix d'où elle tire ses origines. Les analyses devraient couvrir les perspectives des auteurs de violence aussi bien que des victimes, indépendamment de leur sexe.

\section{Abstract}

This article reviews the state of knowledge from studies of sexual violence during armed conflict as well as their different methodologies. It reviews the existing theoretical and empirical literature on this problem, using a mix of qualitative and 
quantitative methods. Findings suggest that "structural" sexual violence is rooted in several social institutions that exacerbate it by transforming it to "warinduced" sexual violence and by impeding prevention and the protection of its victims. Sexual violence leads to physical, psychological and social consequences and may draw its victims into a cycle of violence. Our findings suggest that sexual violence should be monitored in conflict as well as in peace time periods where it is rooted. Analyses should cover the perspectives of perpetrators and victims of both sexes.

Mots clé: Conflit armé, méthodologies, violence sexuelle

\section{Introduction et Importance de L'étude}

La violence sexuelle et le conflit armé sont intimement associés dans l'histoire politique de l'humanité (Denov, 2006; Diken et Lausten, 2005; Watts \& Zimmerman, 2002, Colombini, 2002; Crenshaw, 1991). Néanmoins, les violences sexuelles en période de guerre (VSG) n'ont attiré l'attention soutenue de la communauté internationale qu'à partir des années 1992 et 1994, à la faveur notamment de la médiatisation des cas de violence sexuelle observés au cours des conflits armés en ex-Yougoslavie en 1992 et au Rwanda en 1994. Nordstrom (1994) qualifie ce problème de "secret public" car, ces actes de violence se commettaient au vu d'un public qui gardait le silence. La VSG a longtemps été considérée comme un malheureux effet secondaire de la guerre. Aujourd'hui, la multiplication des informations sur les VSG et la diversité de ces sources - le fait particulièrement des organisations humanitaires, médiatiques et de défense de droits de la personne l - ont attiré l'attention des décideurs politiques internationaux sur les victimes, plus particulièrement les

I. Voir par exemple les sites web suivants: www.un.org, www.watchlist.org, www.unesco.org, www.healafrica.org, www.inrp.fr. femmes et les enfants (Beverly, 1996; Silva, 1994; Catherine, 1995; HRW, 1996). Cependant, du fait même de l'activisme de ces sources et de leur alignement idéologique, leurs analyses n'avancent pas beaucoup la compréhension du phénomène et elles résistent mal aux critiques systématiques.

Au regard de ces descriptions de la violence sexuelle par les organisations internationales et humanitaires depuis les guerres en ex-Yougoslavie et au Rwanda, et en ce début de XXle siècle en République Démocratique du Congo, il n'est pas établi si cette prise de conscience est la conséquence d'une médiatisation internationale ou un éveil de la conscience collective des citoyens dans les milieux où ces violences se commettent. En outre, dans l'état actuel de la recherche sur la violence sexuelle pendant la guerre, nous comprenons encore peu de choses sur les facteurs déterminant l'émergence de ce problème, sa prévention, et ses conséquences. II est ainsi important d'aller au-delà de la simple description et de tenter une analyse des causes profondes. Notre article passe en revue les études réalisées jusqu'à présent sur les VSG afin de guider les études futures. Un revue antérieure (Skjelsbaek 200I) portant sur 140 textes et analysant les 
positions épistémologiques des études antérieures a identifié trois postures principales: l'essentialisme, le structuralisme et le constructivisme social.

Notre revue quant à elle tente de répondre aux deux questions suivantes : que sait-on du phénomène de violence sexuelle en période de guerre? Comment ce phénomène a-t-il été exploré jusqu'à présent? Notre but est de baliser le chemin en terme méthodologiques et substantifs pour d'autres études qui porteront sur les VSG et d'identifier les approches méthodologiques les plus appropriées. Cette étude approfondit l'analyse de Skjelsbaek (200I) en analysant aussi les gestes et intentions des chercheurs tant sur terrain que lors de la rédaction. Ses objectifs spécifiques sont de décrire de manière critique l'état actuel des connaissances sur la VSG par une exploration systématique des études publiées dans les revues ayant un comité de pairs. La focalisation sur ce type d'étude nous permet de nous concentrer sur les travaux les plus rigoureux.

L'importance de cette étude tient à la fois à l'importance du phénomène luimême. Du fait de la multitude et diversité des sources d'informations sur la VSG, la conceptualisation, mesure, et compréhension de ce phénomène demeurent confus. Par conséquent, un des objectifs de notre revue est d'examiner non seulement l'état actuel des connaissances mais aussi leur production. Malgré les multiples conséquences physiques, psychologiques et sociales dues aux violences sexuelles (Kalume et al, 2004) et la mobilisation de nombreux partenaires (étatiques, privés et société civile) autour de ce problème, l'ampleur des VSG semble persister. Notre étude aidera donc les différents partenaires et intervenants à s'attaquer aux causes profondes de ce problème par une approche compréhensive et holistique, et pas seulement s'attaquer aux symptômes.

\section{Méthodologie}

Trois approches méthodologiques sont souvent utilisées dans les revues des études: la méta-analyse, la méta-ethnographie et l'examen systématique de la littérature (Doyle, 2003; Noblit et Hare, 1988). La méta-analyse synthétise les «données» des études quantitatives, parfois en prenant les résultats issus de ces études comme données de départ et en examinant les relations éventuelles entre les conclusions des études et le contexte, les méthodes, ou les caractéristiques des auteurs. La méta-ethnographie synthétise la "substance de la recherche qualitative ". Quant à l'examen systématique de la littérature (Greenhalgh 1997), elle est une vue d'ensemble de recherches ayant utilisé une méthodologie explicite reproductible; par contre, il décrit la méta-analyse comme une synthèse mathématique des résultats d'au moins deux études abordant la même hypothèse de la même manière. La différence entre ces trois méthodologies réside principalement dans le but, la source, la collecte, le processus d'analyse de données, ainsi que le produit final.

S'agissant du but, la méta-analyse vise à synthétiser et accumuler les résultats des études afin de prédire les résultats futurs des problématiques ayant les mêmes conditions. La métaethnographie vise l'explication des études en reconceptualisant les nouvelles interprétations (Doyle, 2003), 
tandis que le but de l'examen systématique de la littérature, selon Krathwahl (1993), est de démontrer comment les études passées sont liées aux théories actuelles et aident à justifier la pertinence des études futures. Les données recherchées dans un examen systématique sont des théories et/ou des conclusions de recherche utilisées pour justifier et orienter les études futures (Glass, 1976). Notre étude se situe dans le contexte de cette dernière approche méthodologique. En effet, nous analysons les études empiriques effectuées sur la VSG afin de bâtir les connaissances futures sur les forces et les faiblesses des études précédentes. Ainsi, nous analysons à la fois des études empiriques quantitatives et qualitatives ayant trait à la violence sexuelle dans un contexte de conflit armé.

Nous avons identifié les articles à travers une recherche systématique des études empiriques dans plusieurs bases de données en utilisant les mots clés suivants : viol, violence sexuelle et guerre ou de manière plus spécifique en anglais «Sex crimes - or - war crime - or rape - or - sexual violence ; war - or armed conflicts." Ces termes devaient se trouver dans le titre, le résumé ou les descripteurs de l'article. Ce processus de recherche à l'aide du thésaurus a permis de repérer un maximum de documents pertinents. La première vague de consultation sur le site de la Bibliothèque de l'Université Laval a per- mis d'identifier plus de 1000 articles tirés des bases de données suivantes: Criminology, Econlit, ERIC, Francis, PAIS International, Pilots Database, Political Science, Psylnfo, Social Services Abstracts, Sociological Abstracts, Sociology, World Wide Political Science Abstracts. Après vérification et tri systématique à partir de titres et d'autres identifiants, 136 articles furent retenus pour notre recherche. Nos critères $d$ ' inclusion des articles furent les suivants: (I) utilisation de données originales sur la violence sexuelle en contexte de conflit armé; (2) revue systématique d'autres études sur la violence sexuelle dans un contexte de conflit armé; (3) publication dans une revue scientifique avec un comité d'évaluation par des pairs; (4) rédaction en anglais ou en français.

Après une première lecture, seulement 6 études sur 136 ont été identifiés comme répondant à tous nos critères d`inclusion. Vu ce faible effectif, nous avons utilisé une technique dite "boule de neige » (Skjelsbaek, 200I) pour identifier plus de textes pertinents. Cette technique consiste à partir d'une référence pour découvrir une autre, lequel nous orientera également vers une autre référence, et ainsi de suite jusqu'à saturation (c'est-à-dire lorsque les articles retenus cessent d'orienter vers de nouvelles références). Finalement, quatorze (14) articles ont ainsi été retenus et examinés (Figure I). 
Figure 1 : Schéma synthétisant le processus de sélection des articles

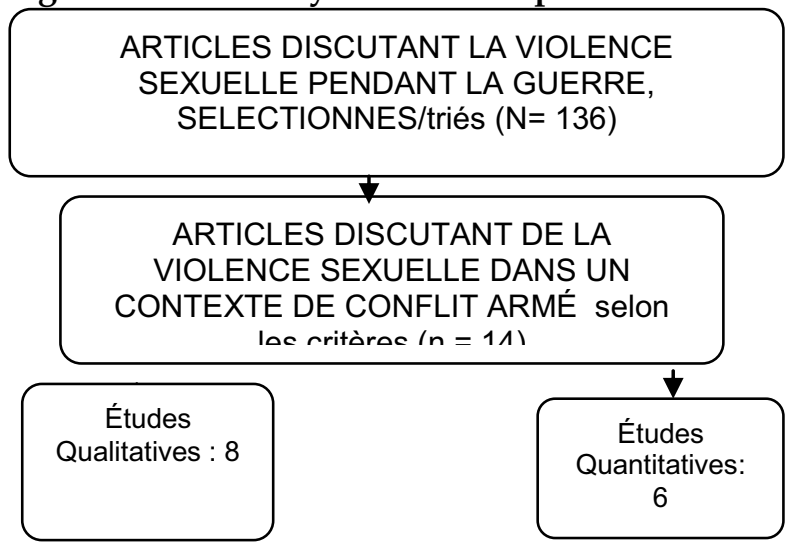

Les données furent enregistrées dans un tableau synthèse comprenant les rubriques suivantes $(I)$ identifiant de l'article (nom de l'auteur ou des auteurs, année de publication, nom du journal et pays où l'étude a été conduite); (2) objectifs de l'étude; (3) méthodes utilisées; (4) résultats; (5) conclusion et recommandations. Ce schéma fut présenté sur une grille permettant d'évaluer une étude afin d'apprécier les liens entre l'objectif ou la question de recherche, la méthodologie utilisée, les résultats obtenus, la conclusion ainsi que les recommandations. La table I résume la distribution des études examinées selon les pays concernés.

Table I Études examinées(14) réparties par pays

\begin{tabular}{llllll}
\hline Ex Yougoslavie $: 03$ & France & $: 01$ & Soudan & $: 01$ \\
Sierra Léone : 03 & États-Unis & $: 01$ & En général & $: 02$ \\
Liberia $:$ 01 & Azerbaijan & $: 01$ & R.D. Congo & $: 00$ \\
El Salvador/ Guatem: 01 & Iraq & $: 01$ & Rwanda & $: 00$ \\
\hline
\end{tabular}

Cette table fait ressortir l'absence quantitative. Nous présenterons nos d'études empiriques en regard des violences sexuelles dans deux pays africains (le Rwanda et la RD Congo) où celles-ci sont généralement considérées comme élevées, selon les différents rapports des organisations humanitaires et les médias internationaux.

\section{Résultats}

De 14 études prises en compte, huit ont utilisé une méthodologie qualitative et six ont utilisé une méthodologie résultats en deux groupes thématiques associées à nos deux questions principales, notamment la méthodologie et l'état des connaissances. En termes de méthodologie, nous avons évalué notamment $(I)$ la collecte et les sources de données et (2) l'évaluation de la qualité des études. En ce qui concerne l'état des connaissances, nous avons fait particulièrement attention aux aspects suivants des VSG: (I) terminologies et définitions; (2) prévalence et incidence; 
(3) victimes et agresseurs; (4) causes et conséquences; (5) protection des victimes et prévention. Les résultats relatifs à ces deux rubriques sont présentés ci-dessous.

\section{Résultats sur la Méthodologie}

L'évaluation de la méthodologie a suggéré les conclusions suivantes:

Collecte et sources des données: La majorité des études recensées reconnaissent que la VSG est un problème complexe en raison des différences dans le contexte, les groupes en conflit, et les intérêts en jeu. Dans l'ensemble, trois principales techniques de collecte de données ont été utilisées: l'entrevue, le questionnaire et la documentation. Seules deux études ont combiné deux techniques: Denov et Maclure, (2006) combinent l'entretien individuel et en groupe tandis que Olujic (1998) combine l'entrevue et le questionnaire. Enfin, une étude a omis de préciser comment les données ont été récoltées. Une première observation s'impose: les techniques de collecte des données utilisées ont peu varié d'une étude à l'autre. Ce manque de diversité dans les techniques de collecte de données peut affecter les résultats et les conclusions des études recensées. En effet, la complexité du problème de violence sexuelle appellerait plutôt, selon nous, à une approche pluridisciplinaire où plusieurs techniques de collecte et sources des données pourraient être combinées pour produire des conclusions plus robustes. Une diversité des techniques devraient également permettre une triangulation des méthodes, des données, des observations et des théories, ce qui conduirait à une interprétation plus riche du phénomène (Angers, 1992). La triangulation devient ainsi une stratégie permettant d'enrichir et de valider une interprétation. Elle vise à établir la concordance entre diverses sources des données et interprétations (La Perrière, 1997 :378).

Les données. Pendant la collecte et la saisie des données, des erreurs comme les doublons, les valeurs manquantes, incomplètes ou peu fiables surviennent fréquemment et elles peuvent influencer les résultats. Sur six études quantitatives, une seule (Swiss et al. 1998), a mentionné comment ces contraintes ont été contournées. A la fin de chaque journée, les membres de l'équipe de l'étude Swiss et al. (1998) devaient vérifier et corriger l'exactitude, la clarté et la complétude de chaque fiche d'enquête. Après que les 205 fiches aient été complétées, la réponse à chaque question d'enquête était transférée sur une fiche de codification permettant ainsi une double-saisie des données dans un programme statistique d'analyse informatisée. Certaines études qualitatives n'ont pas indiqué comment la gestion de la qualité de données a été effectuée, à l'exception de Denov (2006), Denov \& Maclure (2006), Skjelsbaek, (2006) et Olujic, (1998). II faut cependant noter que les exigences des revues scientifiques varient et ceci explique en partie l'omission de ces détails, même si le chercheur en est conscient.

Assurance de la qualité de l'étude. Drapeau (2004:79) souligne que la valeur d'une étude scientifique dépend étroitement de la capacité du chercheur à démontrer la qualité scientifique de ses résultats. Certains procédés permettent d'augmenter la scientificité des résultats des études empiriques. Le plus souvent l'auteur se 
réfère à des critères de scientificité propres à son positionnement épistémologique. Ceux qui utilisent la méthodologie quantitative se réfèrent à des critères statistiques bien précis pour garantir la scientificité de leurs études (Jenicek et Cléroux, 1987). Par ailleurs, Guba et Lincoln (1985) - deux tenants du paradigme naturaliste - ont formulé des critères de crédibilité propres à la recherche qualitative, mais ayant leur équivalent en recherche quantitative (Sabuni, 2004). Ces critères incluent:

la crédibilité (équivalente à la validité interne en recherche quantitative) dont les éléments comprennent la présence prolongée dans le milieu d'étude, l'observation persistante, la triangulation, le débriefing par les pairs, l'analyse des cas négatifs, la vérification par les participants, etc.

la transférabilité (équivalente à la validité externe) qui est la capacité d'une méthodologie de recherche à fournir des résultats généralisables à d'autres populations, à d'autres contextes ou à d'autres périodes. Généralement, elle s'appuie sur trois principes en recherche qualitative : le principe de similitude, c'est-à-dire la possibilité de généraliser les résultats à un univers empirique similaire; le principe de robustesse, selon lequel le potentiel de généralisation s'accroît lorsqu'il y a réplique des effets dans des contextes différents; et enfin le principe d'explication qui est la solidité des concepts et des théories mobilisées pour formuler des hypothèses afin d'interpréter les observations.

la confiance (équivalente à la fiabilité), lorsque le chercheur démontre que le processus de collecte et d'analyse des données était logique, retraça- ble et documenté.

la validation (équivalente à l'objectivité), qui consiste en une description approfondie du contexte avec les participants dans la production des résultats. En d'autres termes, le contexte et les participants déterminent les résultats.

la réflexivité de l'auteur: en tant qu'instrument de recherche, l'auteur doit être conscient et doit démontrer sa capacité à se remettre en question, par exemple, démontrant comment sa position et son background (ses acquis expérientiels et théoriques) peuvent influencer les résultats. II s'agit aussi de la capacité d'explorer des pistes explicatives rivales et de justifier le type de plan de recherche et les choix de méthode de collecte des données, la durée et de l'échantillon.

Denov (2006) et Denov et Maclure (2006) soulignent comment leur présence sur un terrain insécurisé et inhabituel pouvait influencer le processus de récolte de données et comment elles ont géré cette réalité. Skjelsbaek (2006) dans son processus réflexif, reconnaît que l'entrevue avec les femmes violées est problématique, par son éminent caractère politique. En période de conflit armé (souvent sur fond de précarité économique), la présence des personnes et organisations internationales inscrit une autre force (pouvoir) au conflit présent. Bose (2003) nomme cette situation comme étant un nouveau conflit en sourdine, qui engendre des réactions positives ou négatives et affecte le déroulement des entrevues avec les victimes de violence sexuelle. Elle écrit (2006:396) :

"Cela ressort clairement dans leur expression de l'opposition entre eux et nous. Bien que je 
sois une femme et pourrais faire partie du nous femmes, il s'avère que l'on m'attribue davantage l'étiquette du eux, de ces gens provenant de l'international. En conséquence, il est fort probable que les histoires m'ayant été racontées, étaient orientées et colorées de la compréhension qu'avaient ces femmes des acteurs provenant de la communauté internationale -c'est-à-dire des titulaires de pouvoir - désirant connaître les épreuves qu'elles ont endurées. (...). Les protagonistes sont conscients que leurs histoires de viols en période de guerre peuvent être racontées de différentes manières. En d'autres termes, la trame narrative et le contenu du récit peuvent considérablement varier en fonction de l'auditoire. À deux reprises au cours de notre enquête, des participantes nous ont clairement demandé comment nous voudrions que leur histoire nous soit racontée...» (Notre traduction).

Cette capacité du chercheur, de se remettre en question sans compromettre la crédibilité de son étude est importante. Elle se présente comme une chercheuse européenne et consciente de l'apport positif et négatif que son monde, l'Europe, a eu sur la guerre en Bosnie. Par cet exercice de réflexivité, le chercheur maintient et construit sa crédibilité, ce qui, du coup, contribue à la construction du caractère scientifique de l'étude. Enfin, face à la complexité de la question des violences sexuelles en période de conflit armé, les études recensées n'ont pas combiné les deux méthodologies de recherche (quantitative et qualitative). Plutôt que de compter sur une seule approche méthodologique, nous estimons qu'il pourrait être fécond de combiner toutes les données de sources différentes pour construire et analyser la violence sexuelle dans toute sa complexité. L'explication du réel diffère d'une méthodologie à l'autre, tout en se complétant.

\section{Résultats sur l'état des connaissances}

Les principaux résultats sur l'état des connaissances a permis d'aboutir aux conclusions suivantes :

Terminologies et définitions de la VSG. Les termes suivants ont été utilisés dans les différentes études pour désigner, soit la violence sexuelle, soit les différentes formes de violence sexuelle : le viol collectif (Green, 2004), le viol et relation sexuelle forcée (Kerimova et al. 2003; Swiss et al. 1998), les agressions sexuelles liées au régime politique (Amowitz et al. 2004), la violence liée au genre ou sexo-spécifique (Jok Madut, 1999; Denov, 2006), agression sexuelle institutionnalisée et non institutionnalisée (Arons, et al. 1991), viol de guerre (Lilly et Le Roy, 2000), la "pénétration sexuelle " (Diken et Laustsen, 2005). Une seule étude (Green, 2004) a clairement défini sa terminologie - viol collectif - qu'elle décrit comme des viols perpétrés sur des populations civiles par des agents de l'État, des membres de groupe politique ou ethnique. Les études sur la violence sexuelle liée aux conflits armés ou à la présence de personnes en arme n'ont pas utilisé des terminologies standard. 


\section{Prévalence et incidence des VSG}

Six études quantitatives ont analysé la prévalence de la violence sexuelle. Dans les prochaines lignes, nous résumons ces analyses par continent. En Amérique, nomment aux États-Unis, l'étude de Rosen (2007) révèle que la prévalence de la violence sexuelle liée à la présence des hommes en arme dans certains états, avait augmenté entre 1960 et 1992, c'est-à-dire de 8 pour 100.000 à 42 pour 100.000. Entre 1992 et 2000 , elle baissa pour se situer à 33 pour 100.000. Puis, elle augmenta légèrement entre 2000 et 2002 pour se situer à 34,6 pour 100.000 . Un test de corrélation révéla une relation significative entre la prévalence de viol et la proportion du personnel de l'armée de l'air américaine présent pendant la période de 9 ans se situant entre 1994 et 2002. Des analyses plus poussées révélèrent que ces résultats étaient principalement dus à la corrélation élevée entre la présence des forces armées de l'air et d'une population indienne dans certains milieux.

En Afrique, et notamment en Sierra Leone, Amowitz et al. (2002) montre, dans une enquête transversale menée dans trois camps de déplacés internes et dans une ville, que la prévalence cumulée (lifetime prevalence) de violence sexuelle non liée à la guerre, commise par les membres de familles, les amis et la population civile était de $9 \%$. Cette prévalence augmentait à $17 \%$ en additionnant les actes de violence sexuelle directement associée à la guerre. Au Liberia, une autre enquête transversale réalisée dans les camps de déplacés internes, dans les écoles et au marché et dans la ville de Monrovia (Swiss et al. 1998) révéla une prévalence de $49 \%$.
Autrement dit sur 205 femmes et filles âgées de 15 à 70 ans, cent d'entre elles déclarèrent avoir été violées au moins une fois. Cette étude établit un lien significatif entre le fait que des femmes et des filles qui étaient forcées de cuisiner pour les soldats ou les combattants et le fait qu'elles aient affirmé avoir été violée et victime de tentative de viol ou de coercition sexuelle (55\% contre 10\% chez celles n'ayant pas été forcé de cuisiner, $\mathrm{P}<.00 \mathrm{I}, .06, .00 \mathrm{I})$. Enfin, cette étude montre que les jeunes femmes (> 25 ans) avaient plus de chance de reporter le viol ou tentative de viol ou la coercition sexuelle que les femmes âgées ( $<25$ ans) ( $18 \%$ contre $4 \%, P=$ .02 et .04 respectivement).

En Asie, notamment au Sud de l'Iraq, Amowitz et al. (2004) ont étudié la prévalence de la violence sexuelle dans trois grandes villes de neuf gouvernorats (provinces) au sud de l'Iraq. Ils ont trouvé que la prévalence des agressions sexuelles liées au régime politique était de $0,04 \%$, tandis que la prévalence à vie de violence sexuelle liée au régime politique en place était de $5 \%$. Les auteurs de cette recherche estiment que ces deux taux sont inférieurs aux taux reportés à la fois au Sierra Leone, Liberia et aux États-Unis. Ils ajoutent que ceci peut être dû à de fausses réponses négatives liées au stigma social, à la honte et au déshonneur entourant le viol dans la culture musulmane. En Azerbaïdjan, Kerimova et al. (2003) ont constaté que la prévalence des violences sexuelles en période de guerre était de $30 \%$ (soit 135 femmes sur 457). Sur une période de six ans, cette prévalence était de $26 \%$ (soit 120 femmes sur 457) et sur une période d'un an, elle était de $22 \%$ (soit 99 
femmes sur 457).

En somme, la prévalence de violences sexuelles liée aux conflits armés ou en présence de personnes en arme est différente d'une étude à l'autre. Les auteurs ont préféré utiliser le concept de "prévalence" pour décrire la fréquence de violences sexuelles. Nous estimons toutefois qu'une attention soutenue de l'utilisation de ce concept prête à confusion avec le concept d' "incidence », par exemple la prévalence à vie, la prévalence pendant une période de six ans, d'un an...

Victimes et agresseurs. Les principales victimes d'agression sexuelle en période de guerre sont les femmes et les filles dans toutes les études prises en compte. Elles ont été, dans la majorité des études, les participantes/répondantes. Une seule étude (Olujic, 1998) inclut les hommes parmi les participants/répondants, mais pas en tant que victimes. Les agresseurs sont, quant à eux, décrits comme des hommes armés par la majorité des études, sauf une (Denov et Maclure, 2006) qui décrit comment les femmes commandantes (lesbiennes) agressaient les filles soldats au Sierra Leone. Ce qui ressort de ces études, c'est entre autre, la position de faiblesse des victimes en regard de plusieurs facteurs socioéconomiques. Contrairement aux victimes, les agresseurs sont en position de force - ils ont des armes ainsi que d'autres ressources comme la nourriture, qu'ils utilisent comme moyen d'exercer la force ou comme appât pour commettre des violences sexuelles. Sur le terrain des conflits, la possession des armes offre aux agresseurs un pouvoir absolu par rapport aux victimes. Cette relation de pouvoir profondément asymétrique entre les victimes et les agresseurs constitue un élément fondamental dans l'analyse de la violence sexuelle tant en période de conflit armé que pendant la période de paix. Une analyse de cette relation asymétrique des pouvoirs permet de pénétrer la complexité de la problématique de la violence sexuelle en contexte de guerre.

Causes des VSG. Dans notre revue, l'analyse des causes des VSG dans les études qualitatives part généralement du postulat selon lequel la VSG est une constituante indissociable de la guerre (Diken et Laustsen, 2005; Denov, 2006; Denov \& Maclure, 2006). Tout en acceptant ce postulat, d'autres études (Olujic, 1998; Skjelsbaek, 2006; Jok Madut, 1999) démontrent que la violence sexuelle contre la femme ne se limite pas au seul contexte de guerre. Ses racines seraient préétablies en période de paix. Dans ce contexte, l'utilisation de la violence sexuelle contre les femmes en temps de guerre ne peut être comprise sans un examen approfondi de ses significations culturelles en période de paix. Olujic (1998), chercheuse américaine d'origine bosniaque, a analysé la signification de la violence sexuelle dans la culture des Balkans (sud de l'Europe). Elle conclut que dans la culture bosniaque, le « corps individuel de la femme " est quotidiennement, en période de paix, sujet de domination et d'agression - surtout l'essence même de l'être humain - la sexualité et la reproduction. Elle ajoute que pendant la guerre, ce corps "individuel » devient symboliquement un corps « social». Par conséquent, elle conclut que l'utilisation de la violence sexuelle comme "arme de guerre" en ex-Yougoslavie vient directement du concept prévalent Sud- 
Diagramme 1 :

Cadre d'analyse de la violence sexuelle

$=$ relations sexuelles dans un rapport asymétrique des pouvoirs.

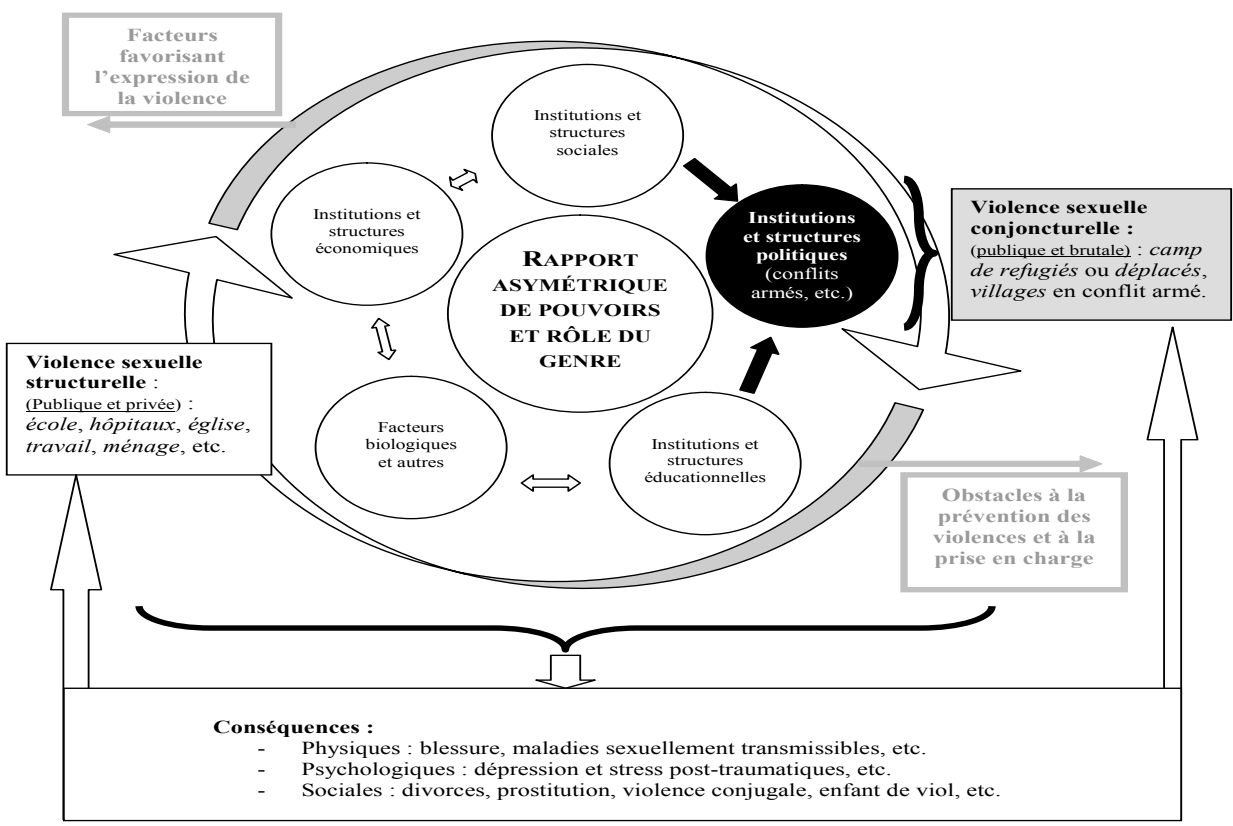

Européen de la sexualité et de l'honneur. Jok Madut (1999), d'origine sud-soudanaise, dans son étude au sud du Soudan, abonde dans le même sens en montrant que la violence sexuelle n'est pas le fait de la violence politique seule, elle est socialement reproduite dans la famille et dans la communauté même en période de paix. Elle distingue ainsi la violence sexuelle dite structurelle (en période de paix) de celle dite conjoncturelle (en période de conflit armé). Nous avons élaboré notre cadre d'analyse (diagramme $I$ ) à partir de cette discussion ainsi que d'autres concepts tirés des études recensées.

Le diagramme I montre comment la violence sexuelle "structurelle » dont les racines sont incarnées dans les institutions et structures sociales, économiques, éducationnelles, peut être exacerbée par les institutions et structures politiques (exemple les forces armées) produisant la violence sexuelle " conjoncturelle ". Ces mêmes institutions peuvent rendre difficile la protection des civiles et la prévention de la violence par un rapport asymétrique des pouvoirs entre et dans le genre. Ces violences ont des conséquences physiques, psychologiques et sociales. Elles peuvent à leur tour replonger les victimes et leurs proches dans le cycle de violences sexuelles par vengeance et toute autre attitude négative. Considérant la complexité de l'analyse de violence sexuelle en contexte de guerre, Skjelsbaek (2006) et Lilly \& Le Roy (2002) nuancent les précédents propos - et estiment qu'il n'est pas productif d'affirmer que la violence sexuelle en période de guerre est 
causée par une hiérarchie dans les relations homme-femme ayant valeur universelle (la patriarchie). En fait, ces auteurs considèrent que cette préconception ne favorise pas l'analyse, parce qu'elle ferme la voie à d'autres stratégies de recherche et d'analyse. Elles proposent donc que l'analyse des causes de la violence sexuelle en contexte de guerre et de leurs significations, tienne compte du lieu, du temps et du groupe dans lequel les violences sont commises afin d'obtenir une vue détaillée du phénomène.

Pour un problème si complexe, les études qualitatives ne peuvent, à elles seules, expliquer toute la profondeur et les dynamiques à l'origine de la genèse des violences sexuelles en contexte de guerre. Toutefois, ces études en offrent un éclairage et des pistes d'analyse spécifiques en s'accordant sur le fait que les violences sexuelles en période de conflit armé (conjoncturelle) trouvent leurs origines au cœur même de la société en période de paix (structurelle).

Une seule étude quantitative (Rosen 2007) a analysé la causalité de la violence sexuelle en période de paix aux États-Unis d'Amérique. Elle suggère que certaines variables soient considérées comme prédicteurs de la violence sexuelle. Par exemple, son étude révèle une corrélation statistiquement significative entre les taux de viol par État, la proportion de personnel de l'armée de l'air dans la population et la proportion de personnes d'origine indienne. L'étude établit aussi une corrélation entre consommation d'alcool par tête d'habitant, taux de viol et présence de personnel des forces aériennes. Enfin, les infections sexuelle- ment transmissibles, en tant que conséquence de violence sexuelle, étaient aussi significativement liées au taux de viol. Toutefois, l'auteur recommande d'autres études plus approfondies pour infirmer ou confirmer ses conclusions.

Conséquences de la VSG. Plusieurs auteurs (Kerimova et al. 2003; Swiss et al. 1998; Amowitz et al. 2002; Amowitz et al. 2004) mentionnent que les maladies sexuellement transmissibles, dont le Sida, sont parmi les conséquences attribuables aux violences sexuelles en contexte de guerre. Aron et al. (199I) décrivent les conséquences psychiques déclenchées par la brutalité avant et pendant la violence sexuelle. Ils démontrent comment les différentes sortes de sévices sexuels engendrent le stress post-traumatiques (SPT) chez les femmes d'Amérique latine refugiées aux États-Unis. Diken et Laustsen (2005) démontrent comment, socialement, la violence sexuelle pendant un conflit armé contribue à détruire la cohésion familiale, la solidarité communautaire et est à la source de la naissance des bébés de la guerre (war babies) par une fécondation forcée (Diken et Laustsen, 2005). Malgré la description des conséquences de violence sexuelle en période de guerre, les auteurs ne sont pas parvenus à mesurer l'ampleur de ces conséquences chez les victimes et dans leurs communautés. II apparait qu'il y a besoin d'explorer l'ampleur des conséquences des violences sexuelles tant en période de paix qu'en période de guerre.

Protection des populations civiles et prévention des VSG. Une seule étude recensée a analysé la protection et la prévention de la violence sexuelle en période de conflit armé. Au 
Sierra-Leone, Denov (2006) évalua le programme de sécurisation et de prévention auprès d'une population civile en période de conflit armé, appelé « Human Security Approach » (HSA). Elle observa que la fin de la guerre au Sierra Leone et la mise en place des programmes tels que le Désarmement, la Démobilisation et la Réinsertion (DDR), la Commission Paix et Réconciliation $(C P R)$ et les Tribunaux, n'ont pas mis un terme aux violences sexuelles. Selon elle, pour offrir à long terme, une plus grande sécurité de la population affectée par les conflits armés, le HSA devrait réformer les structures et les institutions sociales, politiques, économiques et éducationnelles. Denov (2006) poursuit ce raisonnement en mentionnant que, même si le HSA encourage la protection et l'autonomisation des individus, ce programme n'a pas suffisamment abordé les inégalités et les contraintes structurelles qui façonnent la sécurité des filles et des femmes dans un contexte de conflit armé. Cette étude lance donc un défi aux chercheurs et aux décideurs sur la protection effective et la prévention de violence sexuelle en période de conflit armé, car les solutions mises en place jusque là ne touchent pas les causes profondes associées aux violences sexuelles dans la société en général.

\section{Discussion}

Cette étude a tenté de résumer l'état des connaissances et des approches méthodologiques dans la recherche sur les VSG. Sachant que la validité et/ou la crédibilité des résultats est intimement associée à la méthodologie utilisée, nous discuterons de la validité des résultats partant des méthodes uti- lisées par les différents auteurs. Nous discuterons de ces éléments à trois niveaux : (I) les causes, la prévalence et les définitions de la violence sexuelle; (2) la protection et prévention, ainsi que les victimes et agresseurs; (3) les violences sexuelles infligées aux hommes.

\section{Cause, prévalence et terminolo-} gies: Les études quantitatives n'ont pas exploré la causalité des violences sexuelles d'une façon systématique; tout au plus ont-elles décrit la prévalence et l'incidence des viols en période de conflits armés. Cependant, trois études qualitatives indépendantes (Olujic, 1998; Skjelsbaek, 2006; Jok Madut, 1999) ont exploré ce qu'elles appellent les "racines" de la violence sexuelle. Dans leurs analyses, elles ont conclu que la violence sexuelle en période de conflit armé trouve ses « racines» dans la société en période de paix (causes socioculturelles). Elles ajoutent que dans les études de la VSG, il faut éviter de généraliser les conclusions d'une étude à l'ensemble des sociétés. Elles conseillent enfin de prendre en compte le contexte, le temps et le groupe avec lequel les chercheurs travaillent. Skjelsbaek (2006) souligne que c'est ce type d'analyse qui manque aujourd'hui dans les études des phénomènes de VSG.

L'utilisation d'une seule méthode ou d'une seule source de données constitue la faiblesse principale des recherches recensées. Si l'on tient compte de la complexité de la problématique de la violence sexuelle dans un contexte de conflit armé, cela constitue une lacune qu'il faudrait éviter et corriger dans les prochaines études. En dépit de cette faiblesse, la concordance entre diverses 
conclusions des études qualitatives indépendantes apporte une contribution intéressante dans la compréhension de la violence sexuelle en général (Diken et Laustsen, 2005; Denov \& Maclure, 2006; Olujic, 1998; Skjelsbaek, 2006; Jok Madut, 1999). II ressort clairement de ces études que la VSG serait la base de l'iceberg qui ne révèle que sa pointe en période de paix. Ces études permettent ainsi de construire un cadre d'analyse pouvant être utilisé pour des prochaines explorations de la violence sexuelle tant en période de paix qu'en période de conflit armé (voir diagramme I). Cette conclusion des études qualitatives serait plus robuste si elle était triangulée avec des données issues d'études quantitatives de type épidémiologique.

Les auteurs des études quantitatives ont utilisé les termes suivants: prévalence de violence sexuelle liée au régime politique et prévalence à vie. Ces termes prêtent à confusion parce que la période d'étude de ces deux prévalences est différente. En général, l'incidence (ou le taux d'incidence) est le nombre de nouveaux cas d'un phénomène observé pendant une période et pour une population déterminée. Elle est un des critères les plus importants pour évaluer la fréquence et la vitesse d'apparition d'un phénomène. Elle se distingue de la prévalence qui est une mesure d'état (transversale) qui compte tous les cas (nouveaux ou pas) à un moment donné (Jenicek et Cléroux, 1987). Notons enfin que les taux de prévalence de la violence sexuelle révélés dans ces études varient l'une à l'autre. En Sierra Leone, elle est de $9 \%$ dans 3 camps de déplacés; au Liberia, elle est de $9 \%$ aux camps de déplacés internes, dans les écoles, au marché et les environs de la ville de Monrovia. En Iraq, la prévalence (instantanée) est de $0.04 \%$, en Azerbaïdjan, elle est de $30 \%$. Il apparait que ces différences et variations entre les différentes prévalences sont révélatrices des biais.

Premièrement, l'absence d'une définition standard de la violence sexuelle qui est clairement visible dans ces études car chacune utilise ses terminologies. Elles énumèrent plutôt les différentes formes de violence sexuelle plutôt que de les définir, par exemple: viol collectif, relation sexuelle forcée, violence liée au genre, violence sexuelle liée au régime politique, pénétration sexuelle. Deuxièmement, les méthodes utilisées pour identifier la prévalence étaient basées essentiellement sur l'entrevue et/ ou le questionnaire. Considérant les tabous qui entourent la violence sexuelle dans la plupart des sociétés, il est probablement embarrassant de poser une question semblable à celle posée par Kerimova et al. (2003): «A un moment de ta vie, as-tu été forcée par un homme d'avoir une relation sexuelle? Si oui à quel âge?». En plus de l'embarras qu'elle produit chez l'enquêtée, la question a la probabilité d'engendrer de "fausses réponses» par crainte de se voir déshonorer devant des " chercheurs ", qu'ils soient étrangers ou natifs. Le cas de l'étude en Iraq avec Amowitz et al. (2004) en est un exemple typique. Troisièmement, le contexte (lieux) d'enquête est différent d'une étude à l'autre. Pour certaines, le lieu de recherche était les camps de refugiés ou de déplacés, les écoles, les marchés, les villes; pour d'autres, les données ont été recueillies à partir de documents, soit de services judiciaires 
et militaires, soit des medias. Ces différences importantes de contextes rendent difficiles la comparaison et la généralisation des résultats. Enfin, la taille des échantillons dans les diverses études est variée. L'échantillon a varié entre 205 et 199| femmes et le choix des individus n'était pas aléatoire, dû probablement au contexte de guerre. Ces biais d'échantillonnage réduisent la validité interne des études, car, en plus de la petite taille de certaines études, chaque individu n'avait donc pas la même chance de faire partie de l'étude.

Protection et prévention; Victimes et agresseurs. Dans la plupart de contextes de conflits armés, les gouvernements sont sans pouvoir ou inexistants (Nagengast 1994). Ceci peut expliquer en partie que ces gouvernements n'ont pas la main mise sur les agresseurs et n'ont pas non plus une capacité d'aider les victimes (Bose, 2002). Les interventions d'aide aux victimes sont souvent ou toujours apportées par la communauté internationale, coordonnées par les Nations Unies (lbid.). C'est dans ce cadre que les Nations Unies ont élaboré un cadre d'intervention ou programme, appelé la «Human Security Approach» (HSA) qui permet de sécuriser, de protéger et de prévenir les violences de toutes sortes, $y$ compris les violences sexuelles en période de conflits armés. L'évaluation de ce programme par Denov (2006) est pertinente. Fondant son évaluation sur les perspectives et l'expérience de trois filles Sierra Léonaises associées auparavant au groupe armé (RUF), Denov (2006) retrace les formes extrêmes de violence sexuelle et de l'insécurité que les filles ont été forcées de subir, à la fois pendant et après le conflit. Elle examine l'impact de certains programmes de la HSA dont le Désarmement, la Démobilisation et la Réinsertion (DDR), la Commission Paix et Réconciliation (CPR) et les Tribunaux, sur le niveau de l'autonomisation, de la protection et de la sécurité de ces filles. L'auteur conclut que la fin de la guerre et la mise en place de programmes DDR, CPR et Tribunaux n'ont pas signifié la fin de la violence basée sur le genre en Sierra Leone.

Selon elle, même si la HSA encourage la protection et l'autonomisation des individus, elle n'a pas suffisamment abordé les inégalités et les contraintes structurelles qui façonnent la sécurité des filles et des femmes dans un contexte de conflit armé. Elle suggère que pour atteindre une plus grande sécurité à long terme de la population affectée par les conflits, la HSA doit reformer les structures et les institutions sociales, politiques, économiques et éducationnelles. Cette conclusion rejoint, d'une autre façon, la conclusion faite par les autres études qualitatives (Diken et Laustsen, 2005; Denov \& Maclure, 2006; Olujic, 1998; Skjelsbaek, 2006; Jok Madut, 1999), selon lesquelles, la violence sexuelle n'est pas le fait de la violence politique seule, elle est socialement reproduite dans la famille et la communauté. De la même façon que ces structures et institutions sociales, politiques, économiques et éducationnelles favorisent l'incidence de violence sexuelle, de la même façon, ces structures et institutions obstruent la protection et la prévention des victimes de ce type de violence pendant la paix et le conflit armé. En effet, les agresseurs sont souvent protégés par les mythes sociaux, leurs pouvoirs excessifs et la peur 
des victimes, tous ces éléments enracinés dans la culture et la société (Kazawa \& Still, 2000; Lee, 2000; Malinowski, 194I; Roscoe, 1996). Ces structures et institutions pourraient donc entrainer deux effets négatifs contradictoires agissant pour un même but, d'abord en favorisant la violence sexuelle, et ensuite, en bloquant les mesures de protection des individus et la prévention de ces violences dans la société (Abega, 2006).

Scientifiquement, conclure à partir de l'expérience d'un échantillon de trois filles peut sembler inapproprié. Pour ce cas, l'auteur a utilisé un échantillonnage qualitatif appelé "échantillon de cas critique » décrit par Patton (2002). En effet, ces trois filles soldats revêtent une signification particulière dans un contexte de conflit armé, car les filles soldats sont rarement identifiées comme faisant partie de forces combattantes. Ainsi, ces trois "rares" cas de filles soldats peuvent être qualifiés de "rare » et il est permis de croire que les chercheurs auraient pu difficilement accéder à d'autres témoignages.

Omission ou silence sur les victimes « hommes ". Toutes ces études ont généralement reconnu que les victimes les plus fréquentes de la violence sexuelle en période de conflits armés sont les femmes et les filles. Une seule étude a mentionné les hommes comme participants aux enquêtes, mais pas comme victimes. Pourquoi cette omission? II est probable que les hommes victimes taisent cette forme de violence. Cette forme de violence sexuelle faite à l'égard des hommes devra être également prise en compte et comprise si l'on veut comprendre empiriquement l'ensemble du message symbolique que transmettent ceux qui commettent ces crimes. II apparait que nous ne pourrons pas affirmer avoir fait le tour de la question de la violence sexuelle en période de conflits armés tant et aussi longtemps que la dimension des victimes de sexe masculin n'aura pas également été prise en compte.

\section{Conclusion}

Les VSG sont un problème mieux reconnu depuis les guerres de l'ex-Yougoslavie et du Rwanda. Elles ont souvent été perçues comme une tactique de guerre délibérée ayant pour but l'humiliation, la domination, le terrorisme et la dispersion de la population civile. Limiter ces analyses à ce niveau ne permet pas de prendre des mesures de prévention et de protection adéquates. Notre étude ouvre de nouveaux horizons en révélant que la VSG peut être le prolongement de la violence sexuelle dans la société pendant la période de paix. Pourtant actuellement, les intervenants et bailleurs de fonds ici la communauté internationale - mettent l'accent exclusivement ou presque sur la violence sexuelle observées en période de conflits armés. II apparait que pour mieux comprendre la violence en période de guerre, il est mieux d'explorer ce phénomène au sein des sociétés en période de paix. Même en période de paix, le ventre de la femme et/ou le sexe en général incarne un objet d'exercice de domination et d'humiliation. II apparait aussi que la littérature sur la violence sexuelle en période de conflits armés souffrira d'un manque de rigueur si elle n'intègre pas également l'analyse de la violence sexuelle perpétrée sur les hommes. Reconnaître que la violence sexuelle 
faite aux hommes en période de conflits armés ne peut en rien minimiser l'importance de la violence faite aux femmes et les relations asymétriques des pouvoirs et des rôles entre l'homme et la femme. II s'agit plutôt de rendre compte de la réalité et faire le tour complet de la question de violence sexuelle en période de conflits armés. II nous apparaît urgent d'entreprendre des études et des analyses approfondies de la violence sexuelle dans les milieux publics (écoles, milieux de travail, hôpitaux, marchés, etc.) aussi bien que dans les milieux dit privés comme le ménage, en période structurelle où la violence sexuelle se manifeste. Finalement, mentionnons que les méthodologies et les méthodes de recherche devront être contextualisées, diversifiées, rigoureuses et inclure tous les éléments qui influencent l'asymétrie sociale du pouvoir à tous les niveaux des classes sociales.

\section{Références}

Abega, S.C.2006. La violence endémique en Afrique, Bulletin de I'APAD, No 25, La violence endémique en Afrique, (en ligne) http://apad.revues.org/org/ document $199 . \mathrm{html}$, consulté le 28 février 2008.

Angers, M. 1992. Initiation pratique à la méthodologie des sciences humaines. Anjou, Centre éducatif et culturel : 365p.

Amowitz, L.L., Reis, C., Lyons, K.H., Vann, B. Mansaray, B. AkinsulureSmith, A.M., Taylor, L., lacopino, V. 2002. "Prevalence of war-related sexual violence and other human rights abuses among internally displaced persons in Sierra Leone"
JAMA, 287 (4):5 I3-52I.

Amowitz, L.L., Kim, G., Reis, C., Asher, J.L., lacopino, V. 2004. "Human rights abuses and concerns about women's health and human rights in South Iraq" JAMA, 29I(I2): 147I-9.

Aron, A., Corne, S. Fursland, A., Zelwer, B. 1991. "The genderspecific terror of El Salvador and: post-traumatic stress disorder in Central American Refugee Women" Women's Studies International Forum, 14(1/2):37-47.

Beverly, A. 1996. Rape Warfare: The Hidden Genocide in BosniaHerzegovina and Croatia. Minneapolis: University of Minnesota Press.

Bose, S. 2002. Bosnia After Dayton:

Nationalist Partition and International Intervention. New York: Oxford University Press.

Catherine, N.N. 1995. "Women, War and Rape: Challenges Facing the International Tribunal of the Yugoslavia" Human Rights Quarterly, (I7):649-90

Colombini, M. 2002. "Gender-based and Sexual Violence Against Women during Armed Conflict" Journal of Health Management, 4(2): 167-I83

Crenshaw, K. 1991. "Mapping the margins: Intersectionality, identity politics, and violence against women of color" Stanford Law Review, 43: I24I-I 299.

Denov. M.S. 2006. "Wartime sexual violence: assessing a Human Security Response to war-affected girls in Sierra Leone" Security Dialogue, 37(3):319-342.

Denov, M. \& Maclure, R. 2006. "Engaging the voices of the girls in the aftermath of Sierra Leone's 
conflict: experiences and perspectives in a culture of violence" Anthropologica, 48: I - 3.

Diken, B. \& Laustsen C.B. 2005. "Becoming abject: rape as weapon of war" Body Society, I I (I): I I I-I 28.

Drapeau, M. 2004. "Les critères de scientificité en recherche qualitative" Pratiques psychologiques, (I0): 79-86.

Doyle, L.H. 2003. "Synthesis through meta-ethnography: paradoxes, enhancements, and possibilities" Qualitative Research 3(3): 32I-344.

Glass, G.V. 1976. "Primary, Secondary, and Meta-analysis Research" Educational Researcher 5(10): 3 - 8.

Gohier, C. 2004. "De la démarcation entre critères d'ordre scientifique et d'ordre éthique en Recherche interprétative" Recherches Qualitatives, 24 : 3-17.

Green, J.L (2004) "Uncovering Collective Rape: a comparative study of political sexual violence" International Journal of Sociology, 34(I):97 - I I6.

Greenhalgh, T. 1997. "How to read a paper: Papers that summarise other papers (systematic reviews and meta-analyses)" BMJ, 13(3I5):672675.

Guba, E. \& Lincoln, Y. 1994. "Competing paradigms in qualitative research". In N. Denzin \& Y. Lincoln (Eds), Handbooks of qualitative research (pp. 105-II7) Thousand Oaks, CA: Sage.

(HRW) Human Rights Watch 1996.

Shattered Lives: Sexual Violence During the Rwandan Genocide and its Aftermath. New York: Human Rights Watch.

Jenicek, M. et Cléroux, R. 1987.
Épidémiologie: Principes, Techniques, Applications. $4^{\mathrm{eme}}$ Edition. ÉdisemMaloine $454 \mathrm{p}$.

Jok Madut, J. 1999. "Militarisation and gender violence in South Soudan" JAAS, 34(4):427-442.

Kalume, M.A., Ahuka, O. Sergio C. Stone, Lyn L., Lusi, K.M., Bola, M. 2004. La violence sexuelle à l'est de la RDC en période de conflit: particularités et conséquences. 3(I3) : II76-II82.

Kazawa S. \& Still M.C. 2000. "Why Men Commit Crimes (And Why They Desist)" Sociological Theory, 8 (3): 434-447

Kerimova, J., Posner, S.F., Brown, T., Hillis, S., Meikle, S., Duerr A. 2003. "High prevalence of self-reported forced sexual intercourse among internally displaced in Azerbaijan" American Journal of Public Health, 93(7): 1067-70.

Krathwahl, D.R. 1993. Methods of

Educational and Social Science Research: An Integrated Approach. New York: Longman

La perrière, A. 1997. "Les critères de scientificité des méthodes qualitatives". Dans "La recherche qualitative: enjeux épistémologiques et méthodologiques». Sous la direction de Jean Poupart, Boucherville, Gaëtan Morin: pp. 365-389.

Lee M.R. 2000. "Community Cohesion and Violent Predatory Victimisation: A Theoretical Extension and CrossNational Test of Opportunity Theory" Social Forces, 79 (2) : 683706

Lilly J.R. \& Le Roy F. 2002. "'Larmée américaine et les viols en France, Juin 1944 - mai 1945" Revue 
d'histoire, 75(numéro spécial): p. III.

Malinowski,

B. 1941.

"An

Anthropological Analysis of War", The American Journal of Sociology, 46 (4): $521-550$

Nagengast, C. 1994. "Violence, Terror and the Crisis of the State" Annual Review of Anthropology, (23): 109136.

Noblit, G.W. and Hare, R.D. 1988. Meta-ethnography: Synthesizing Qualitative Studies. Newbury Park, CA: Sage Publications.

Nordstrom, C. 1994. Rape: Politics and Theory in War and Peace. Working Paper 146. Canberra: Peace Research Centre, Australian National University.

Olujic M.B. 1998. "Embodiment of terror: gendered violence in peacetime and wartime in Croatia and Bosnia-Herzegovina" Medical Anthropology Quarterly, I2(I): 3I-50.

Patton, M.Q. 2002. Qualitative research and

evaluation methods ( $3^{\text {rd }}$ Ed.) Thousands Oakes, CA: Sage Publications.

Roscoe, P.B. 1996. "War and Society in Sepik New Guinea" The Journal of the Royal Anthropological Institute, 2 (4): 645-666.

Rosen, L.N. 2007. "Rape rates and military personnel in the United States: an exploratory study" Violence Against Women, 13(9): 945-
960.

Sabuni, L.P. 2004. "Gender differences in attitudes and practices within households in rural areas of the Democratic Republic of Congo" These de Doctorat, Université de Liverpool, RU.

Silva, M. 1994. "Gender as an EthnoMarker: Rape, War and Identity in the Former Yugoslavia, in Valentine", M. Moghadam (ed.) Identity Political and Women: Cultural Reassertion and Feminisms in International Perspective, s. 76-97. Boulder: Westview Press.

Skjelsbaek, I. 2000. "Sexual Violence and War: Mapping Out a Complex Relationship" European Journal of International Relations, 7(2):21 I-27.

Skjelsbaek, I. 2006. "Victim and survivor: narrated social identities of women who experienced rape during the war in BosniaHerzegovina" Feminist \& Psychology, 16(4):373-403

Swiss, S., Jennings, P.J., Aryee, G.V., Brown, G.H., Jappah-Samukai, R.M., Kamara, M.S., Schaak, D.H. 1998. "Violence against women during the Liberian civil conflict" American Medical Associations, 279:625-629

Watts, C. \& Zimmerman, C. (2002). "Violence against women: global scope and magnitude" The Lancet, 359: I232-1237. 